Georgian Mathematical Journal

1(94), No. 4, 405-417

\title{
ON SOME GENERALIZATIONS OF THE VANDERMONDE MATRIX AND THEIR RELATIONS WITH THE EULER BETA-FUNCTION
}

\author{
I. LOMIDZE
}

\begin{abstract}
A multiple Vandermonde matrix which, besides the powers of variables, also contains their derivatives is introduced and an explicit expression of its determinant is obtained. For the case of arbitrary real powers, when the variables are positive, it is proved that such generalized multiple Vandermonde matrix is positive definite for appropriate enumerations of rows and columns. As an application of these results, some relations are obtained which in the one-dimensional case give the well-known formula for the Euler betafunction.
\end{abstract}

It is known from the classical problem of multiple interpolation (cf. [1], pp. 104-106; [2], pp. 13-16) that there exists the unique polynomial $P_{N-1}$ of degree $N-1$ which satisfies the conditions

$$
P_{N-1}^{(k-1)}\left(x_{j}\right)=y_{j}^{(k-1)}, \quad k=\overline{1, r_{j}}, \quad j=\overline{1, n},
$$

where $x_{j}, y_{j}^{(k-1)}$ are given elements of an arbitrary field $F ; x_{j} \neq x_{l}$ if $j \neq l, r_{j} \geq 1$ and $N=\sum_{j=1}^{n} r_{j}$. Hence the determinant of the $N \times N$ matrix $V\left(x_{1}, r_{1} ; \ldots ; x_{n}, r_{n}\right) \in M_{N}(F)$ is nonzero. Here by $M_{l}(F)$ with natural $l$ we denote the set of all $l \times l$ matrices with elements from $F$ and by $V\left(x_{1}, r_{1} ; \ldots ; x_{n}, r_{n}\right)$ the $N \times N$ block-matrix

$$
V\left(x_{1}, r_{1} ; \ldots ; x_{n}, r_{n}\right)=\left[w_{1}\left(x_{1}\right)\left|w_{2}\left(x_{2}\right)\right| \cdots \mid w_{n}\left(x_{n}\right)\right],
$$

where $w_{j}, j=\overline{1, n}$, stand for the rectangular matrices

$$
w_{j}(x)=\left[\left(x^{i-1}\right)^{(k-1)}\right]_{\substack{1 \leq i \leq N \\ 1 \leq k \leq r_{j}}}
$$

1991 Mathematics Subject Classification. 15A15, 15A54, 33B15. 
There are other cases when one has to deal with matrices of the form (2). For instance, the determinant of such a matrix can be effectively used when proving the proposition on functional independence of elements from the complete set of invariants of operators (no matter whether they are Hermitian or not) in an $N$-dimensional unitary space if these operators have $r_{j}$-dimensional eigenspaces, $r_{j}>1$ (this proposition will be published in forthcoming paper of the author). The case $r_{j}=1$ has been investigated in [3].

For $r_{1}=\cdots=r_{n}=1$ we have $N=n$ and (2) becomes the Vandermonde matrix $V=V\left(x_{1}, \ldots, x_{n}\right) \in M_{n}(F)$

$$
V\left(x_{1}, 1 ; \ldots ; x_{n}, 1\right)=\left[x_{j}^{i-1}\right]_{1}^{n}
$$

with the determinant

$$
\operatorname{det}\left[x_{j}^{i-1}\right]_{1}^{n}=\prod_{1 \leq k<j \leq n}\left(x_{j}-x_{k}\right) .
$$

1. In what follows $\mathbb{N}=\{1,2, \ldots\}, F$ stands for the field of real numbers $\mathbb{R}$ or that of complex numbers $\mathbb{C}$ and we shall call $(2)$ the $\left(r_{1}, \ldots, r_{n}\right)$-tiple Vandermonde matrix. For $r_{1}=\cdots=r_{n}=r$ this matrix is said to be the $r$-tiple Vandermonde matrix and we denote it by $V\left(x_{1}, \ldots, x_{n} ; r\right)$.

Theorem 1. For arbitrary $r_{1}, r_{2}, \ldots, r_{n} \in \mathbb{N}$ the $\left(r_{1}, r_{2}, \ldots, r_{n}\right)$-tiple Vandermonde matrix (2) with $x_{1}, \ldots, x_{n} \in F$ satisfies the identity

$$
\operatorname{det} V\left(x_{1}, r_{1} ; \ldots ; x_{n}, r_{n}\right)=\left(\prod_{j=1}^{n} \prod_{k=0}^{r_{j}-1}(k !)\right) \prod_{1 \leq i<k \leq n}\left(x_{k}-x_{i}\right)^{r_{k} r_{i}} .
$$

In particular,

$$
\operatorname{det} V\left(x_{1}, \ldots, x_{n} ; r\right)=\left(\prod_{k=0}^{r-1}(k !)\right)^{n} \prod_{1 \leq i<k \leq n}\left(x_{k}-x_{i}\right)^{r^{2}} .
$$

Before proving Theorem 1 we need some preparation.

Lemma 1. Let $n, N \in \mathbb{N}, 1 \leq n \leq N$ and $\phi_{j k}, f_{i}: F \rightarrow F$ be such that

$$
\begin{aligned}
\phi_{j k} \in C^{k-1}(F, F), \quad k=\overline{1, r_{j}}, \quad j=\overline{1, n}, \\
f_{i} \in C^{r-1}(F, F), \quad r=\max \left\{r_{j} \mid j=\overline{1, n}\right\}, \quad i=\overline{1, N}, \quad N=\sum_{j=1}^{n} r_{j},
\end{aligned}
$$

and let $U \in M_{N}(F)$ be the square $N \times N$ block-matrix

$$
U=\left[u_{1}\left(x_{1}\right)|\cdots| u_{n}\left(x_{n}\right)\right],
$$


where

$$
u_{j}(x)=\left[\left(f_{i}(x) \phi_{j k}(x)\right)^{(k-1)}\right]_{\substack{1 \leq i \leq N \\ 1 \leq k \leq r_{j}}}, x \in F .
$$

Then we have

$$
\operatorname{det} U=\operatorname{det}\left[v_{1}\left(x_{1}\right)|\cdots| v_{n}\left(x_{n}\right)\right] \prod_{j=1}^{n} \prod_{k=1}^{r_{j}}\left(\phi_{j k}\left(x_{j}\right)\right)
$$

with

$$
v_{j}(x)=\left[\left(f_{i}(x)\right)^{(k-1)}\right]_{\substack{1 \leq i \leq N \\ 1 \leq k \leq r_{j}}}
$$

Proof of Lemma 1. Let us denote by $[k]_{j}$ the column having number $k$ in the matrix $u_{j}, k=\overline{1, r_{j}}, j=\overline{1, n}$. It is clear that (8) is trivial if $\phi_{j 1}\left(x_{j}\right)=0$ for some $j, 1 \leq j \leq n$. Now if $\phi_{j 1}\left(x_{j}\right) \neq 0$ for each $j=\overline{1, n}$, then it is sufficient to show that by elementary transformations of the columns of (7) it is possible to reduce them to the form

$$
[k]_{j} \rightarrow[\widetilde{k}]_{j}=\left[\left(f_{i}(x)\right)^{(k-1)} \phi_{j k}(x)\right]_{1 \leq i \leq N}
$$

This will be proved by induction. The column $[1]_{j}$ in (7) already has the desired form (9) $[\widetilde{1}]_{j}=[1]_{j}=\left[f_{i}(x) \phi_{j 1}(x)\right]_{1 \leq i \leq N}, j=\overline{1, n}$.

Let us assume that the columns $\left\{[k]_{j} \mid k=\overline{1, m}\right\}, 1 \leq m \leq r_{j}-1$, also have the desired form (9) for each $j, j=\overline{1, n}$. If now $\phi_{j k}\left(x_{j}\right)=0$ for some $k, 1 \leq k \leq m$, then (8) is proved. Next, if

$$
\phi_{j k}\left(x_{j}\right) \neq 0
$$

for each $j, k, j=\overline{1, n}, k=\overline{1, m}$, then we add the sum

$$
\sum_{q=1}^{m} C_{m}^{q-1}\left(-\phi_{j, m+1}^{(m-q+1)}(x) / \phi_{j q}(x)\right)[q]_{j}=\sum_{q=0}^{m-1} C_{m}^{q}\left(-\phi_{j, m+1}^{(m-q)}(x)\right) f_{i}^{(q)}(x)
$$

(which is correctly defined according to $(10)$ ) to the column $[m+1]_{j}$ written by virtue of the Leibniz formula as

$$
[m+1]_{j}=\left[\left(f_{i}(x) \phi_{j, m+1}(x)\right)^{(m)}\right]_{1 \leq i \leq N}=\left[\sum_{q=0}^{m} C_{m}^{q} f_{i}^{(q)}(x) \phi_{j, m+1}^{(m-q)}(x)\right]_{1 \leq i \leq N} .
$$

Finally we obtain

$$
[m+1]_{j} \rightarrow[\widetilde{m+1}]_{j}=\left[f_{i}^{(m)}(x) \phi_{j m+1}(x)\right]_{1 \leq i \leq N} .
$$


Proof of Theorem 1. Denote by $\{i\}$ the row with the number $i, i=\overline{1, N}$, in the matrix (2). By adding the sum $\sum_{q=1}^{r_{1}} c_{q}\{q+i-1\}$ with the coefficients $c_{q} \in F$ to the row $\left\{r_{1}+i\right\}, i=\overline{1, N-r_{1}}$, we get

$$
\operatorname{det} V\left(x_{1}, r_{1} ; \ldots ; x_{n}, r_{n}\right)=\operatorname{det}\left[\begin{array}{c:c|c}
\widetilde{w}_{1}\left(x_{1}\right) & & \widetilde{w}_{n}\left(x_{n}\right) \\
\hdashline- & \cdots & - \\
\widetilde{u}_{1}\left(x_{1}\right) & & \widetilde{u}_{n}\left(x_{n}\right)
\end{array}\right],
$$

where the matrix $\widetilde{w}_{j}(x), j=\overline{1, n}$, is obtained from the matrix (3) after eliminating the last $N-r_{1}$ rows, and the matrix $\widetilde{u}_{j}(x), j=\overline{1, n}$, has the form

$$
\widetilde{u}_{j}(x)=\left[\left(x^{i-1} Q(x)\right)^{(k-1)}\right]_{\substack{1 \leq i \leq N-r_{1} \\ 1 \leq k \leq r}} .
$$

Here

$$
Q(x)=x^{r_{1}}+\sum_{q=1}^{r_{1}} c_{q} x^{q-1}
$$

is a polynomial of degree $r_{1}$.

Note that the matrix $\widetilde{w}_{1}(x)=V\left(x, r_{1}\right)$ is the $r_{1} \times r_{1}$ Wronski matrix of $r_{1}$ functions $\left\{f_{i}(x)=x^{i-1} \mid i=\overline{1, r_{1}}\right\}$ and therefore

$$
\operatorname{det} V\left(x, r_{1}\right)=\operatorname{det}\left[\widetilde{w}_{1}(x)\right]=\prod_{k=0}^{r_{1}-1} k !
$$

Combining the coefficients $c_{q} \in F, q=\overline{1, N-n}$, in such a way that $Q$ has the $r_{1}$-tiple root at $x=x_{1}$, we obtain

$$
\begin{gathered}
Q(x)=\left(x-x_{1}\right)^{r_{1}}, \\
Q^{(k)}\left(x_{1}\right)=0, \quad k=\overline{0, r_{1}-1},
\end{gathered}
$$

and now the matrix $\widetilde{u}_{1}\left(x_{1}\right)$ vanishes by virtue of the Leibniz formula and (12). Therefore

$$
\operatorname{det} V\left(x_{1}, r_{1} ; \ldots ; x_{n}, r_{n}\right)=\operatorname{det}\left[\begin{array}{c:c:c|c}
\widetilde{w}_{1}\left(x_{1}\right) & \widetilde{w}_{2}\left(x_{2}\right) & & \widetilde{w}_{n}\left(x_{n}\right) \\
\hdashline- & - & \ldots & \widetilde{u}_{2}\left(x_{2}\right) \\
0 & & \widetilde{u}_{n}\left(x_{n}\right)
\end{array}\right],
$$

which, taking into account (13) and the Laplace theorem on determinant expansion, gives

$$
\operatorname{det} V\left(x_{1}, r_{1} ; \ldots ; x_{n}, r_{n}\right)=\left(\prod_{k=0}^{r_{1}-1} k !\right) \operatorname{det} \widetilde{U},
$$


where the matrix $\widetilde{U} \in M_{N-r_{1}}(F), \widetilde{U}=\left[\widetilde{u}_{2}\left(x_{2}\right)|\cdots| \widetilde{u}_{n}\left(x_{n}\right)\right]$, satisfies the conditions of Lemma 1. Hence according to (8) and (3)

$$
\operatorname{det} \widetilde{U}=\operatorname{det}\left[w_{2}\left(x_{2}\right)|\cdots| w_{n}\left(x_{n}\right)\right] \prod_{j=2}^{n}\left(Q\left(x_{j}\right)\right)^{r_{j}}
$$

and from (16) together with (2) and (14) we obtain a recurrence relation

$$
\begin{gathered}
\operatorname{det} V\left(x_{1}, r_{1} ; \ldots ; x_{n}, r_{n}\right)= \\
\left(\prod_{k=0}^{r_{1}-1} k !\right) \prod_{j=2}^{n}\left(x_{j}-x_{1}\right)^{r_{1} r_{j}} \operatorname{det} V\left(x_{2}, r_{2} ; \ldots ; x_{n}, r_{n}\right) .
\end{gathered}
$$

The induction by $n$ gives (5) from (17) and (13).

2. Let the reals $\alpha_{i}, x_{j}, i, j=\overline{1, n}$, be given. We introduce the notation $(\alpha)_{n}=\left\{\alpha_{i} \mid \alpha_{i} \neq \alpha_{j}\right.$ if $\left.i \neq j, \quad i=\overline{1, n}\right\}$.

It is known that the generalized Vandermonde matrix

$$
\begin{gathered}
{\left[x_{j}^{\alpha_{i}}\right]_{1}^{n}=V^{(G)}\left(x_{1}, \ldots, x_{n} ;(\alpha)_{n}\right) \equiv V^{(G)}} \\
\left(0<x_{1}<\cdots<x_{n} ; \alpha_{1}<\alpha_{2}<\cdots<\alpha_{n}\right)
\end{gathered}
$$

is completely positive (cf.[4], p.372), i.e., all its minors are positive.

Let now $1 \leq n \leq N$ and $r_{1}, r_{2}, \ldots, r_{n} \in \mathbb{N}$. We shall call the matrix

$$
V^{(G)}\left(x_{1}, r_{1} ; \ldots ; x_{n}, r_{n} ;(\alpha)_{N}\right)=\left[w_{1}^{(G)}\left(x_{1}\right)|\cdots| w_{n}^{(G)}\left(x_{n}\right)\right]
$$

the $\left(r_{1}, r_{2}, \ldots, r_{n}\right)$-tiple generalized Vandermonde matrix. Here $w_{j}^{(G)}, j=$ $\overline{1, n}$, is the rectangular matrix

$$
w_{j}^{(G)}(x)=\left[\left(x^{\alpha_{i}}\right)^{(k-1)}\right]_{\substack{1 \leq i \leq N \\ 1 \leq k \leq r_{j}}}, \quad\left(r_{j} \geq 1, j=\overline{1, n}, \sum_{j=1}^{n} r_{j}=N\right) .
$$

Theorem 2. The $\left(r_{1}, r_{2}, \ldots, r_{n}\right)$-tiple generalized Vandermonde matrix

$$
\begin{gathered}
V^{(G)}\left(x_{1}, r_{1} ; \ldots ; x_{n}, r_{n} ;(\alpha)_{N}\right) \\
\left(0<x_{1}<\cdots<x_{n} ; \alpha_{1}<\alpha_{2}<\cdots<\alpha_{n}\right)
\end{gathered}
$$

is positively definite.

Lemma 2. For arbitrary simultaneously nonzero reals $c_{i} \in \mathbb{R}, i=\overline{1, N}$, $\alpha_{i} \neq \alpha_{k}$ if $i \neq k$, the function $f: \mathbb{R} \rightarrow \mathbb{R}$

$$
f(x)=\sum_{i=1}^{N} c_{i} x^{\alpha_{i}}
$$


has at most $N-1$ positive zeros counted according to their multiplicities:

$$
\begin{gathered}
f(x)=\prod_{j=1}^{n}\left(x-x_{j}\right)^{r_{j}} \phi\left(x ;(\alpha)_{N}\right), \\
\sum_{j=1}^{n} r_{j} \leq N-1 ; \phi\left(x ;(\alpha)_{N}\right) \neq 0, \quad x>0 ; \quad x_{j}>0, \quad j=\overline{1, n}, \quad 1 \leq n \leq N .
\end{gathered}
$$

Proof of Lemma 2. If $f$ has only single zeros, i.e., $r_{1}=\cdots=r_{N}=1$, then $n=N$, and the assertion of Lemma 2 follows from the inequality $\operatorname{det} V^{(G)} \neq 0$ ([4], p.372). Assume that the assertion of Lemma 2 is true for $1 \leq r_{j} \leq r, j=\overline{1, n}$, and prove it for $N$ power-summands also in the case $r_{j^{\prime}}=r+1$ for certain $j^{\prime}, 1 \leq j^{\prime} \leq n$.

Let the opposite be true: say, there exist reals $c_{i}, i=\overline{1, N}$, at least one of which is nonzero, such that

$$
\begin{gathered}
f(x)=\sum_{i=1}^{N} c_{i} x^{\alpha_{i}}=\prod_{j=1}^{n}\left(x-x_{j}\right)^{r_{j}} \phi\left(x ;(\alpha)_{N}\right), \\
\sum_{j=1}^{n} r_{j} \geq N ; \quad 1 \leq r_{j} \leq r+1, \quad j=\overline{1, n}, \quad 1 \leq n \leq N .
\end{gathered}
$$

Let $m, 1 \leq m \leq n$, be the number of multiple zeros of $f$ and zeros $x_{j}$, $j=\overline{1, n}$, be arranged according to the decrease of their multiplicity. Then $f$ satisfies the conditions

$$
\begin{gathered}
f\left(x_{j}\right)=0, \quad j=\overline{1, n} \\
f^{\prime}\left(x_{j}\right)=\cdots=f^{\left(r_{j}-1\right)}\left(x_{j}\right)=0, \quad 2 \leq r_{j} \leq r+1, \quad j=\overline{1, m}
\end{gathered}
$$

here

$$
n-m+\sum_{j=1}^{m} r_{j} \geq N .
$$

The Rolle theorem, together with (20), implies that the function $\tilde{f}$ : $\mathbb{R} \rightarrow \mathbb{R}$

$$
\widetilde{f}(x)=\left(f(x) x^{-\alpha_{1}}\right)^{\prime}=\sum_{i=1}^{N-1} \widetilde{c}_{i} x^{\alpha_{i}}
$$

vanishes at the points $\xi_{j}, 0<x_{j}<\xi_{j}<x_{j+1}, j=\overline{1, n-1}$, i.e.,

$$
\widetilde{f}\left(\xi_{j}\right)=0, \quad j=\overline{1, n-1} .
$$


Moreover, from (21) it follows that

$$
\widetilde{f}\left(x_{j}\right)=\widetilde{f}^{\prime}\left(x_{j}\right)=\cdots=\widetilde{f}^{\left(r_{j}-2\right)}\left(x_{j}\right)=0, \quad j=\overline{1, m},
$$

i.e., $\widetilde{f}$ has the form

$$
\begin{gathered}
\widetilde{f}(x)=\prod_{k=1}^{n-1}\left(x-\xi_{k}\right) \prod_{j=1}^{m}\left(x-x_{j}\right)^{r_{j}-1} \widetilde{\phi}\left(x ;(\widetilde{\alpha})_{N}\right)= \\
=P_{N}^{\sim}(x) \widetilde{\phi}\left(x ;(\widetilde{\alpha})_{n}\right),
\end{gathered}
$$

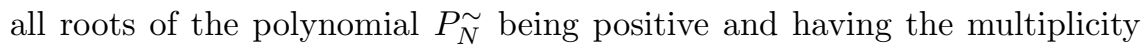
$\leq r$. From (24) and (22) we find

$$
\widetilde{N}=n-1+\sum_{j=1}^{m}\left(r_{j}-1\right)=n-1+\sum_{j=1}^{m} r_{j}-m \geq N-1,
$$

but this contradicts our assumption, since the function (23) is the sum of $N-1$ power-summands.

Proof of Theorem 2. For $\alpha_{i}=i-1, i=\overline{1, N}$, we have

$$
V^{(G)}\left(x_{1}, r_{1} ; \ldots ; x_{n}, r_{n} ;(\alpha)_{N}\right)=V\left(x_{1}, r_{1} ; \ldots ; x_{n}, r_{n}\right)
$$

and, according to (5),

$$
\begin{gathered}
\operatorname{det} V^{(G)}\left(x_{1}, r_{1} ; \ldots ; x_{n}, r_{n} ;(\alpha)_{N}\right)>0 \\
\left(0<x_{1}<\cdots<x_{n} ; \quad \alpha_{i}=i-1, \quad i=\overline{1, N}\right) .
\end{gathered}
$$

It is possible to pass to arbitrary values $\alpha_{1}<\cdots<\alpha_{N}$ starting from the integers $\alpha_{i}=i-1, i=\overline{1, N}$, and changing them continuously, but preserving the inequalities among them. In doing so, the determinant $\operatorname{det} V^{(G)}\left(x_{1}, r_{1} ; \ldots ; x_{n}, r_{n} ;(\alpha)_{N}\right)$ does not vanish according to Lemma 2 and therefore for all $0<x_{1}<\cdots<x_{n} ; \alpha_{1}<\alpha_{2}<\cdots<\alpha_{N}$ we have $\operatorname{det} V^{(G)}\left(x_{1}, r_{1} ; \ldots ; x_{n}, r_{n} ;(\alpha)_{N}\right)>0$.

Since each principal minor of the matrix $V^{(G)}\left(x_{1}, r_{1} ; \ldots ; x_{n}, r_{n} ;(\alpha)_{N}\right)$ can be considered as a determinant of a certain $\left(r_{1}, r_{2}, \ldots, r_{n}\right)$-tiple generalized Vandermonde matrix, all such minors are positive.

Corollary 1. It follows from $\operatorname{det} V^{(G)}\left(x_{1}, r_{1} ; \ldots ; x_{n}, r_{n} ;(\alpha)_{N}\right) \neq 0$ that for arbitrary reals $\alpha_{i}, \alpha_{i} \neq \alpha_{k}$ if $i \neq k, i, k=\overline{1, N}$, there exists unique collection of reals $c_{i}, i=\overline{1, N-1}$, such that

$$
\sum_{i=1}^{N-1} c_{i} x^{\alpha_{i}}+x^{\alpha_{n}}=P_{N-1}(x) \phi\left(x ;(\alpha)_{N}\right),
$$


where $P_{N-1}$ is any given polynomial of degree $N-1$ having only positive roots, and $\phi\left(x ;(\alpha)_{N}\right) \neq 0$ for $x>0$.

Note that for $\alpha_{i} \in \mathbb{N}$ the above proposition is nothing but the Viete theorem, since it enables us to determine the coefficients at power-summands provided we know zeros of the sum.

3. Let $n, N$ be integers, $1 \leq n \leq N$, and $0<\alpha_{1}<\alpha_{2}<\cdots<$ $\alpha_{N}, x_{j}>0, j=\overline{1, n}$. Transform the determinant $\operatorname{det} V^{(G)}\left(x_{1}, r_{1} ; \ldots ;\right.$ $\left.x_{n}, r_{n} ;(\alpha)_{N}\right)$, taking the factor $\alpha_{i}, i=\overline{1, N}$, out of the $i$-th row and taking into account that

$$
\alpha_{i}^{-1}\left(x^{\alpha_{i}}\right)^{(k-1)}=\frac{\Gamma\left(\alpha_{i}\right)}{\Gamma\left(\alpha_{i}+2-k\right)} x^{\alpha_{i}-k+1}=\left(x^{\alpha_{i}-1}\right)^{(k-2)}, \quad k=1,2, \ldots .
$$

Here we put

$$
\begin{gathered}
\left(x^{\alpha}\right)^{(-q)}=\underbrace{\int_{0}^{x} d x \cdots \int_{0}^{x}}_{q-\text { tiple }} d x x^{\alpha}=\frac{1}{\Gamma(q)} \int_{0}^{x}(x-t)^{q-1} t^{\alpha} d t, \\
\quad q=1,2, \ldots, \\
\left(x^{\alpha}\right)^{(-1)}=\int_{0}^{x} t^{\alpha} d t, \quad \alpha \geq 0, \quad x>0,
\end{gathered}
$$

and $\Gamma(z)=(z-1)$ ! is the gamma-function. Taking into account the corollary of Theorem 2, after the appropriate transformations we get

$$
\begin{gathered}
\operatorname{det} V^{(G)}\left(x_{1}, r_{1} ; \ldots ; x_{n}, r_{n} ;(\alpha)_{N}\right)= \\
=(-1)^{\sigma_{1}} \prod_{i=1}^{N} \alpha_{i} \operatorname{det} V^{(G)}\left(x_{1}, r_{1}-1 ; \ldots ; x_{n}, r_{n}-1 ;(\alpha-1)_{N-n}\right) \operatorname{det} A
\end{gathered}
$$

where

$$
\sigma_{1}=\sum_{j=1}^{n} j\left(r_{j}-1\right), \quad(\alpha-1)_{N}=\left\{\alpha_{i}-1 \mid \alpha_{i} \neq \alpha_{j} \text { if } i \neq j, i, j=\overline{1, N}\right\},
$$

and $A \in M_{n}(F)$ is a square matrix of the form

$$
A=\left[a_{i j}\right]=\left[\int_{x_{j-1}}^{x_{j}} \phi_{i}\left(t ;(\alpha-1)_{\widetilde{N}}\right) \prod_{p=1}^{n}\left(t-x_{p}\right)^{r_{p}-1} d t\right] .
$$

Here we use the notation

$$
\widetilde{N}=N-n+1
$$


and $\phi_{i}(t ;(\alpha-1) \widetilde{N})$ is defined by the formula

$$
\phi_{i}\left(t ;(\alpha-1) \widetilde{N}_{\tilde{N}}\right) \prod_{p=1}^{n}\left(t-x_{p}\right)^{r_{p}-1}=\sum_{q=0}^{N-n} c_{i+q} t^{\alpha_{i+q}-1}, \quad i=\overline{1, n},
$$

the coefficients $c_{i+q}$ being defined according to the corollary of Theorem 2 .

Rearranging the columns of $\operatorname{det} V^{(G)}\left(x_{1}, r_{1} ; \ldots ; x_{n}, r_{n} ;(\alpha)_{N}\right)$ which contain higher derivatives, we can reduce it to the form

$$
\begin{gathered}
\operatorname{det} V^{(G)}\left(x_{1}, r_{1} ; \ldots ; x_{n}, r_{n} ;(\alpha)_{N}\right)= \\
=(-1)^{\sigma_{2}} \operatorname{det} V^{(G)}\left(x_{1}, r_{1}-1 ; \ldots ; x_{n}, r_{n}-1 ;(\alpha)_{N-n}\right) \operatorname{det} \widetilde{A},
\end{gathered}
$$

where $\sigma_{2}=\sum_{j=1}^{n}(j-1)\left(r_{j}-1\right)$ and the matrix $\widetilde{A} \in M_{n}(F)$ is written as

$$
\begin{gathered}
\widetilde{A}=\left[\widetilde{a}_{i j}\right]_{1}^{n}=\left[\left(\widetilde{\phi}_{i}(x ;(\alpha) \widetilde{N}) \prod_{p=1}^{n}\left(x-x_{p}\right)^{r_{p}-1}\right)_{\mid x=x_{j}}^{\left(r_{j}-1\right)}\right]_{1}^{n}= \\
=\left[\left(r_{j}-1\right) ! \widetilde{\phi}_{i}\left(x_{j} ;(\alpha) \widetilde{N}\right) \prod_{\substack{p=1 \\
p \neq j}}^{n}\left(x_{j}-x_{p}\right)^{r_{p}-1}\right]_{1}^{n} .
\end{gathered}
$$

Here, by definition,

$$
\widetilde{\phi}_{i}\left(x ;(\alpha) \widetilde{N}_{p}\right) \prod_{p=1}^{n}\left(x-x_{p}\right)^{r_{p}-1}=\sum_{q=0}^{N-n} c_{i+q} x^{\alpha_{i+q}}, \quad i=\overline{1, n},
$$

from which one can find

$$
\widetilde{\phi}_{i}\left(x ;(\alpha)_{\widetilde{N}}\right)=x \phi_{i}\left(x ;(\alpha-1)_{\widetilde{N}}\right), \quad i=\overline{1, n} .
$$

Equating the right-hand sides of (26) and (30) and taking into account (31) and (32), we get

$$
\begin{gathered}
\frac{\operatorname{det}\left[\int_{x_{j-1}}^{x_{j}} \phi_{i}(t ;(\alpha-1) \widetilde{N}) \prod_{\substack{p=1 \\
p \neq j}}^{n}\left(\frac{t-x_{p}}{x_{j}-x_{p}}\right)^{r_{p}-1}\left(x_{j}-t\right)^{r_{j}-1} d t\right]_{1}^{n}}{\operatorname{det}\left[\phi_{i}\left(x_{j} ;(\alpha-1) \widetilde{N}_{1}\right)\right]_{1}^{n} \prod_{p=1}^{n} x_{j}}= \\
=\prod_{j=1}^{n}\left(r_{j}-1\right) ! \prod_{i=1}^{N} \alpha_{i}^{-1} \frac{\operatorname{det} V^{(G)}\left(x_{1}, r_{1}-1 ; \ldots ; x_{n}, r_{n}-1 ;(\alpha)_{N}\right)}{\operatorname{det} V^{(G)}\left(x_{1}, r_{1}-1 ; \ldots ; x_{n}, r_{n}-1 ;(\alpha-1)_{N}\right)} . \\
\left(x_{p} \neq x_{j} \quad \text { if } \quad p \neq j ; \quad x_{0}=0 ; \quad i, j=\overline{1, n}\right) .
\end{gathered}
$$

For the right-hand side ratio Lemma 1 obviously gives

$$
\frac{\operatorname{det} V^{(G)}\left(x_{1}, r_{1}-1 ; \ldots ; x_{n}, r_{n}-1 ;(\alpha)_{N-n}\right)}{\operatorname{det} V^{(G)}\left(x_{1}, r_{1}-1 ; \ldots ; x_{n}, r_{n}-1 ;(\alpha-1)_{N-n}\right)}=\prod_{j=1}^{n} x_{j}^{r_{j}-1} \text {. }
$$


Hence we have the formula

$$
\begin{gathered}
\frac{\operatorname{det}\left[\int_{x_{j-1}}^{x_{j}} \phi_{i}\left(t ;(\alpha-1)_{\widetilde{N}}\right) \prod_{\substack{p=1 \\
p \neq j}}^{n}\left(\frac{t-x_{p}}{x_{j}-x_{p}}\right)^{r_{p}-1}\left(x_{j}-t\right)^{r_{j}-1} d t\right]_{1}^{n}}{\operatorname{det}\left[\phi_{i}\left(x_{j} ;(\alpha-1) \widetilde{N}\right)\right]_{1}^{n} \prod_{p=1}^{n} x_{j}^{r_{j}}}= \\
=\frac{\prod_{p=1}^{n}\left(r_{p}-1\right) !}{\prod_{i=1}^{N} \alpha_{i}} \\
\left(x_{p} \neq x_{j} \text { if } p \neq j ; r_{p} \in \mathbb{N}, \sum_{p=1}^{n} r_{p}=N, x_{p}>0, p, i, j=\overline{1, n} ; x_{0}=0\right),
\end{gathered}
$$

which can be rewritten as

$$
\begin{gathered}
\frac{\operatorname{det}\left[\int_{x_{j-1} / x_{j}}^{1}(1-u)^{r_{j}-1} \phi_{i}\left(u x_{j} ;(\alpha-1)_{\widetilde{N}}\right) \prod_{\substack{p=1 \\
p \neq j}}^{n}\left(\frac{x_{j} u-x_{p}}{x_{j}-x_{p}}\right)^{r_{p}-1} d u\right]_{1}^{n}}{\operatorname{det}\left[\phi_{i}\left(x_{j} ;(\alpha-1)_{\widetilde{N}}\right)\right]_{1}^{n}}= \\
=\frac{\prod_{k=1}^{n} \Gamma\left(r_{k}\right)}{\prod_{i=1}^{N} \alpha_{i}} \\
\left(x_{p} \neq x_{j} \text { if } p \neq j ; r_{p} \in \mathbb{N}, \sum_{p=1}^{n} r_{p}=N, x_{p}>0, p, i, j=\overline{1, n} ; x_{0}=0\right) .
\end{gathered}
$$

In the particular case where $\alpha_{i}=r_{0}-1+i, r_{0}>0, i=\overline{1, N}$, we have

$$
\begin{aligned}
& \phi_{i}\left(t ;(\alpha-1)_{\widetilde{N}}\right)=\left(\prod_{p=1}^{n}\left(t-x_{p}\right)^{r_{p}-1}\right)^{-1} \sum_{q=0}^{N-n} c_{i+q} t^{r_{0}-1+i+q-1}= \\
& =t^{r_{0}+i-2}\left(\prod_{p=1}^{n}\left(t-x_{p}\right)^{r_{p}-1}\right)^{-1} \sum_{q=0}^{N-n} c_{i+q} t^{q}=t^{r_{0}+i-2}, \quad i=\overline{1, N} .
\end{aligned}
$$

The latter equality is valid because, according to the Viete theorem, there exist reals $c_{i+q}$ such that

$$
\sum_{q=0}^{N-n} c_{i+q} t^{q}=\left(\prod_{p=1}^{n}\left(t-x_{p}\right)^{r_{p}-1}\right)
$$

Moreover,

$$
\prod_{i=1}^{N}\left(r_{0}-1+i\right)=\prod_{i=1}^{N} \alpha_{i}=\frac{\Gamma\left(r_{0}+N\right)}{\Gamma\left(r_{0}\right)}=\frac{\Gamma\left(r_{0}+\sum_{p=1}^{n} r_{p}\right)}{\Gamma\left(r_{0}\right)}
$$


and (33) transforms to the equality

$$
\begin{gathered}
\frac{\operatorname{det}\left[x_{j}^{i-1} \int_{x_{j}-1 / x_{j}}^{1} u^{r_{0}+i-2}(1-u)^{r_{j}-1} \prod_{\substack{p=1 \\
p \neq j}}^{n}\left(\frac{x_{j} u-x_{p}}{x_{j}-x_{p}}\right)^{r_{p}-1} d u\right]_{1}^{n}}{\operatorname{det}\left[x_{j}^{i-1}\right]_{1}^{n}}= \\
=\frac{\prod_{p=0}^{n} \Gamma\left(r_{p}\right)}{\Gamma\left(\sum_{p=0}^{n} r_{p}\right)} \\
\left(x_{p} \neq x_{j} \text { if } p \neq j ; \quad r_{p} \in \mathbb{N}, \quad x_{p}>0, \quad p, i, j=\overline{1, n} ; \quad x_{0}=0, \quad r_{0}>0\right) .
\end{gathered}
$$

For the case $n=1$ the formula (34) gives the well-known expression

$$
\int_{0}^{1} u^{r_{0}-1}(1-u)^{r_{1}-1} d u=\frac{\Gamma\left(r_{0}\right) \Gamma\left(r_{1}\right)}{\Gamma\left(r_{0}+r_{1}\right)}=B\left(r_{0}, r_{1}\right)
$$

for the Euler integral of the first kind.

Now, we introduce the notation

$$
\begin{gathered}
B_{n}(\mathbf{r})=B\left(r_{0}, r_{1}, \ldots, r_{n}\right)= \\
=\left\{\begin{array}{l}
1, \quad n=0, \\
\operatorname{det}\left[x_{j}^{i-1} \int_{x_{j-1} / x_{j}}^{1} u^{i-1}(1-u)^{r_{j}-1} \prod_{\substack{k=0 \\
k \neq j}}^{n}\left(\frac{x_{j} u-x_{k}}{x_{j}-x_{k}}\right)^{r_{k}-1} d u\right]_{1}^{n} / \operatorname{det}\left[x_{j}^{i-1}\right]_{1}^{n}, \\
n \geq 1 \quad\left(x_{p} \neq x_{j} \text { if } p \neq j, p, j=\overline{0, n}, x_{0}=0\right)
\end{array}\right.
\end{gathered}
$$

for arbitrary $\left\{r_{j} \mid r_{j}>0, j=\overline{0, n}\right\} \equiv \mathbf{r}$.

Since the Euler formula (35) is valid for arbitrary complex $r_{0}, r_{1}, \operatorname{Re} r_{0}>$ $0, \operatorname{Re} r_{1}>0$, there arises the problem:

Problem. Is the equality

$$
B_{n}(\mathbf{r})=B\left(r_{0}, r_{1}, \ldots, r_{n}\right)=\frac{\prod_{j=0}^{n} \Gamma\left(r_{j}\right)}{\Gamma\left(\sum_{j=0}^{n} r_{j}\right)}
$$

fulfilled for an arbitrary complex $r_{j}, j=\overline{0, n}, n \geq 2$ ? (Note that this case is not covered either by (34) or by (35)).

4. Put $2 \leq n \leq N$ and $1 \leq m \leq \min \left\{r_{j} \mid j=\overline{1, n}\right\}$. Transform the determinant on the left-hand side of $(5)$, taking the factor $\Gamma(i) / \Gamma(i-m)$ out of each row having the number $i \geq m+1$ and keeping in mind that

$$
\begin{gathered}
\left(\frac{\Gamma(i-m)}{\Gamma(i)} x^{i-1}\right)^{(k-1)}=\frac{\Gamma(i-m)}{\Gamma(i-k+1)} x^{i-k}=\left(x^{i-m-1}\right)^{(k-m-1)} \\
(i=\overline{m+1, N}, \quad 1 \leq m \leq \min \{r \mid j=\overline{1, n}\}, \quad k=1,2, \ldots) .
\end{gathered}
$$


After easy but rather long calculations, using (25) and (5) we get the formula

$$
\frac{\operatorname{det}\left[B^{(1)}|\ldots| B^{(n)}\right]}{\operatorname{det} V\left(x_{1}, \ldots, x_{n} ; m\right)}=\prod_{k=1}^{m}\left(\frac{\Gamma(k)}{\Gamma(N-k+1)} \prod_{j=0}^{n} \Gamma\left(r_{j}-k+1\right)\right),
$$

where $B^{(j)}, j=\overline{1, n}$, is a rectangular matrix of the form

$$
\begin{gathered}
B^{(j)}=\left[b_{i k}^{(j)}\right]_{\substack{1 \leq i \leq m n \\
1 \leq k \leq m}}= \\
=\left[x_{j}^{i-k} \int_{x_{j-1} / x_{j}}^{1}(1-u)^{r_{j}-m} u^{k+i-2} \prod_{\substack{p=0 \\
p \neq j}}^{n}\left(\frac{x_{j} u-x_{p}}{x_{j}-x_{p}}\right)^{r_{p}-m} d u\right]_{\substack{1 \leq i \leq m n \\
1 \leq k \leq m}} \\
\left(x_{p} \neq x_{j} \text { if } p \neq j ; \quad x_{0}>0 ; \quad j=\overline{1, n}\right) .
\end{gathered}
$$

In particular, for $m=1$ (38) reduces to (34).

5. Let us now apply the above results to the case $n=1, N=r, x>0$. It is easy to show that for the determinant of the matrix

$$
V^{(G)}\left(x, r ;(\alpha)_{r}\right)=\left[\left(x^{\alpha_{i}}\right)^{(k-1)}\right]_{1}^{r},
$$

which is nothing but the Wronski matrix for $r$ functions $\left\{f_{i}(x)=x^{\alpha_{i}} \mid i=\right.$ $\overline{1, r} ; x>0\}$, one gets

$$
\operatorname{det} V^{(G)}\left(x, r ;(\alpha)_{r}\right)=x^{\beta} \prod_{1 \leq k<j \leq r}\left(\alpha_{j}-\alpha_{k}\right),
$$

where $\beta=\sum_{i=1}^{N} \alpha_{i}-r(r-1) / 2$.

Obviously, the formula (40) generalizes (13).

Using (40), one can find values of $\phi\left(x ;(\alpha)_{N}\right)$ and coefficients $c_{i}$ for which

$$
\sum_{i=1}^{N-1} c_{i} x^{\alpha_{i}}+x^{\alpha_{N}}=\left(x-x_{1}\right)^{N-1} \phi\left(x ;(\alpha)_{N}\right) .
$$

Namely, the Cramer formulas give

$$
\begin{gathered}
c_{i}=-x_{1}^{\alpha_{N}-\alpha_{i}} L_{i}\left(\alpha_{N}\right), \quad i=\overline{1, N-1} \\
\phi\left(x ;(\alpha)_{N}\right)=\left(x-x_{1}\right)^{-(N-1)} x_{1}^{\alpha_{N}}\left(\left(x / x_{1}\right)^{\alpha_{N}}-\sum_{i=1}^{N-1}\left(x / x_{1}\right)^{\alpha_{i}} L_{i}\left(\alpha_{N}\right)\right),
\end{gathered}
$$

where

$$
L_{i}(\alpha)=\prod_{\substack{p=1 \\ p \neq i}}^{N-1}\left(\frac{\alpha-\alpha_{p}}{\alpha_{i}-\alpha_{p}}\right), \quad i=\overline{1, N-1}
$$


is the Lagrange elementary interpolation polynomial. (41) gives

$$
\begin{gathered}
\phi\left(x_{1} ;(\alpha-1) \widetilde{N}\right)= \\
=\frac{x_{1}^{\alpha_{N}-N}}{(N-1) !}\left(\frac{\Gamma\left(\alpha_{N}\right)}{\Gamma\left(\alpha_{N}-N+1\right)}-\sum_{i=1}^{N-1} \frac{\Gamma\left(\alpha_{i}\right)}{\Gamma\left(\alpha_{i}-N+1\right)} L_{i}\left(\alpha_{N}\right)\right), \\
\phi\left(u x_{1} ;(\alpha-1) \widetilde{N}\right)=\frac{x_{1}^{\alpha_{N}-N}}{(u-1)^{(N-1)}}\left(u^{\alpha_{N}-1}-\sum_{i=1}^{N-1} u^{\alpha_{i}-1} L_{i}\left(\alpha_{N}\right)\right) .
\end{gathered}
$$

Substituting (42) and (43) in (33) for $n=1, N=r_{1} \geq 2, \widetilde{N}=N$ and performing integration we obtain

$$
\begin{gathered}
\frac{\Gamma\left(\alpha_{n}\right)}{\Gamma\left(\alpha_{N}-N+1\right)}-\sum_{i=1}^{N-1} \frac{\Gamma\left(\alpha_{i}\right)}{\Gamma\left(\alpha_{i}-N+1\right)} L_{i}\left(\alpha_{N}\right)= \\
=(-1)^{N}\left(\sum_{i=1}^{N-1} \alpha_{i}^{-1} L_{i}\left(\alpha_{N}\right)-\alpha_{N}^{-1}\right) \prod_{k=1}^{N} \alpha_{k} .
\end{gathered}
$$

Finally, substitution of (44) in (42) gives

$$
\phi\left(x_{1} ;(\alpha-1)_{\widetilde{N}}\right)=\frac{x_{1}^{\alpha_{N}-N}}{(N-1) !}(-1)^{N}\left(\sum_{i=1}^{N-1} \alpha_{i}^{-1} L_{i}\left(\alpha_{N}\right)-\alpha_{N}^{-1}\right) \prod_{k=1}^{N} \alpha_{k} .
$$

\section{REFERENCES}

1. R. W. Hamming, Numerical methods for scientists and engineers. (Translation into Russian) Mir, Moscow, 1968; English original: McGrawHill, 1962.

2. N.A.Lebedev and V.I.Smirnov, The constructive theory of functions of a complex variable. (Russian) Nauka, Moscow, 1964.

3. I.R.Lomidze, Criteria of unitary and orthogonal equivalence of operators. Bull. Acad. Sci. Georgia 141(1991), No. 3, 481-483.

4. F.R.Gantmakher, The theory of matrices. (Russian) Nauka, Moscow, 1988.

(Received 24.11.1992)

Author's address:

Faculty of Physics

I. Javakhishvili Tbilisi State University

3, I. Chavchavadze Ave, 380028 Tbilisi

Republic of Georgia 